\title{
Must Hidden VARIABLES THeORIES Be CONTEXTUAL? KOCHEN \& SPECKER MEET VON NEUMANN AND GLEASON ${ }^{1}$
}

\author{
Pablo ACUÑa \\ Philosophy Institute \\ Pontifical Catholic University of Chile \\ ptacuna@uc.cl
}

\begin{abstract}
It is a widespread belief that the Kochen-Specker theorem imposes a contextuality constraint on the ontology of beables in quantum hidden variables theories. On the other hand, after Bell's influential critique, the importance of von Neumann's wrongly called 'impossibility proof' has been severely questioned. However, Max Jammer, Jeffrey Bub and Dennis Dieks have proposed insightful reassessments of von Neumann's theorem: what it really shows is that hidden variable theories cannot represent their beables by means of Hermitian operators in Hilbert space. Hereby I show that $i$ ) the very same constraint can be derived from Gleason's theorem, and that ii) if we consider the import of von Neumann's and Gleason's theorems, the relevance of the Kochen-Specker theorem for hidden variables theories gets substantially weakened: it does not force them to be contextual in any interesting sense of the term.
\end{abstract}

\section{INTRODUCTION}

Since the very formulation of quantum mechanics in the early decades of the $20^{\text {th }}$ century, physicists and philosophers got involved in discussions about what is the description of the physical world that the theory offers. According to the Copenhagen interpretation, which became dominant by the 1930s, the descriptive content of quantum mechanics reaches only observed reality, whereas a postulated objective reality independent of measurements is simply beyond its grasp, or even beyond scientific meaningfulness. Some notable figures like Einstein, de Broglie and Schrödinger, resisted this view and defended that there is indeed an objective quantum reality, and that the theory does not capture it because it is not the whole story. This line of resistance to the Copenhagen interpretation constitutes the basis for the 'hidden variables program'. The basic idea in this program is that if suitable extra variables are added to the mathematical machinery of quantum mechanics, the theory would be able to describe the physical world independently of observation and measurement. That is, the hidden variables would come to supply the description of features of physical systems that the quantum formalism misses. ${ }^{2}$

\footnotetext{
${ }^{1}$ European Journal for Philosophy of Science 11, 41 (https://doi.org/10.1007/s13194-021-00347-8). Please refer to the published version for citation purposes.

2 The attitude that the great dissidents took towards hidden variables is a complex issue. Einstein was critical of Bohm's (1952) theory (see Myrvold 2003). Furthermore, in 1927 he sketched a hidden variables theory, but he did not publish the manuscript and abandoned the project-his discontent seems to have been non-locality (see Belousek 1996; Holland 2005). De Broglie formulated a hidden variables theory in 1927 (very similar to Bohm's), but he soon abandoned it after criticisms he received during the Fifth Solvay Conference. However, he reconverted to the hidden variables view after Bohm's (1952) work (see Bacciagaluppi \& Valentini 2009). Schrödinger defended the incompleteness view, but he pointed out formal constraints on viable hidden variables theories that prefigure von Neumann's theorem. He took these constraints as serious obstacles, but his precise attitude towards the program is not entirely clear (see Bacciagaluppi \& Crull 2009).
} 
Two formal results played important roles in the historical development and evaluation of the program. The first one, introduced by John von Neumann (1955) in 1932, was taken as a plain proof of the impossibility of a completion of quantum mechanics with hidden variables, or at least as a strong advice against the development of the project. Historical accounts disagree about the extent and nature of vNT's impact, but it certainly contributed to at least discourage the interest in hidden variables theories (HVTs). ${ }^{3}$

Three decades later, John Bell (1966) strongly criticized von Neumann's result: he showed that it is not an impossibility proof, and he further argued that the theorem is rather trivial: according to Bell, it only rules out an uninteresting and irrelevant class of HVTs. In 1935, Grete Hermann (2016) had presented a somewhat similar argument, but it did not have much impact in the community. The core of Bell's and Hermann's criticism is that a premise assumed by von Neumann cannot be justified for physically plausible HVTs. Unlike Hermann's, Bell's negative assessment of vNT became very influential, and, to this day, it constitutes a common opinion in the philosophy and foundations community.

In the same paper, Bell (1966) obtained another formal result, and Simon Kochen and Ernst Specker (1967) independently proved the same statement one year later. The Kochen-Specker theorem $(\mathrm{KST})^{4}$ is generally understood as imposing some kind of contextuality constraint on the ontology of physical quantities (properties, beables) 5 in HVTs. That is, this theorem is typically interpreted as establishing that the ontology of beables portrayed by these theories must be such that, for some properties, either the possessed values or their very empirical effectiveness must depend on the context of measurement.

Considering the basic spirit and goals of HVTs, this is a substantial compromise. If true, ontological contextuality involves a significant concession to Copenhagen style quantum mechanics, insofar as measurement-independent values for contextual properties become impossible. Furthermore, since the assumption that Hermann and Bell criticize in vNT plays no role in the KST, the general opinion in the philosophy and foundations community is that the latter is a much more important result than the former for HVTs (see, for example, Held 2018).

In this article, I contest the prevailing interpretation of both theorems. Despite general disdain, vNT does teach us something fundamental about the nature of HVTs. Jeffrey Bub $(2010,2011)$ has shown that what the theorem really states is that the beables in these theories cannot be represented by Hermitian operators. Max Jammer (1974) proposed an analysis of vNT that prefigures Bub's, and Dennis Dieks (2017) defends a reading of the theorem along Bub's line. Here I will show that the

\footnotetext{
${ }^{3}$ Some accounts state that vNT was understood as a crucial factor for a disdainful attitude towards hidden variables in general, and towards Bohm's theory (1952) in particular-see, for example, Cushing (1994) and Whitaker (1996). This view has been contested - see, for example, Dieks (2017) and Myrvold (2003). I remain neutral about this historical-sociological controversy. What is at stake here is the precise conceptual import of vNT.

4 The right name would be "Bell-Kochen-Specker theorem". However, the most common tag in the literature is "Kochen-Specker theorem". This label is unfair, but at least it prevents confusion with Bell's inequalities theorem. For simplicity, I will stick to the usual name.

${ }^{5}$ I will use the term "observable" in a literal way, that is, as referring to some quantity observed in a measurement, independently of the ontological status of what is measured. That is, remaining agnostic as to whether the measured quantity belongs to the measured system or to the system-and-apparatus, as to whether the quantity has objective reality independently of measurements or not, etc. I reserve the term "beable" for the usual notion of property possessed by a physical system, independently of measurements.
} 
very same lesson can be extracted from an unquestioned and celebrated theorem introduced by Andrew Gleason (1957). That is, my first claim is that Gleason's theorem (GT), just like vNT, proves that in viable HVTs beables cannot be represented by Hermitian operators, but now on the basis of premises that are immune to the Hermann-Bell criticism.

Second, I argue that if we read the KST in the light of vNT and GT, we see that the former does not really force HVTs to be contextual - at last not in the usual ontological reading of 'contextual' so it does not really oblige them to make any important concessions in their basic motivation. As we will see, the reason is that the contextual-ontology reading of the KST crucially assumes that in HVTs Hermitian operators represent the theory's beables, but this is forbidden by vNT and GT.

In short, I claim that the customary understanding of the KST for the ontology of HVTs must be reassessed, and that the usual judgment about the relevance of the theorems is upside down. That is, vNT (and also GT) imposes a deeper and more significant constraint on HVTs than the rather weak restriction that the KST really enforces. Actually, the latter does not compel viable HVTs to be contextual, in any interesting, or peculiarly quantum, sense of the term.

In section 2, I present the KST, and I explain why it is generally interpreted as implying that the ontology of properties in HVTs must be contextual. In section 3, I focus on vNT. First, I present its logical structure, then Bell's critique, and finally Jammer's and Bub's lucid reassessments. In section 4, I show that GT proves the same result obtained by von Neumann. The upshot is that both theorems prove that HVTs within Hilbert space-in which beables are represented by Hermitian operators-are not possible. In section 5, I first show that if we consider the restriction that both vNT and GT impose on HVTs, we realize that the KST has no bearing on the ontology of the beables in such theories, for the alleged ontological contextuality constraint crucially presupposes that beables in HVTs are indeed represented by Hermitian operators. Then, I clarify the real import of the KST for HVTs in the light of vNT and GT, illustrating with the case of Bohmian Mechanics, and comparing my proposal with Bell's (1966) take on the KST. In section 6, I discuss an argument introduced by Meyer (1999), Kent (1999) and Clifton \& Kent (2000) that intends to "nullify" the import of the KST. In section 7, I summarize and conclude.

\section{THE KOCHEN-SPECKER THEOREM}

The KST is well-known in the philosophy and foundations of physics community. I will present just the essential elements that motivate its usual interpretation, i.e., that it imposes a contextuality constraint on the ontology of beables in HVTs. In subsections 2.1 and 2.3, I follow the structure of Michael Redhead's (1987) analysis. Redhead's examination is very useful for our purposes. That the KST forces the beables in HVTs to be contextual is indeed a common belief, but it is not always precisely articulated. Redhead's analysis makes that statement very clear. Besides, it is a good representative of the usual interpretation-e.g., the entry on the KST in the Stanford Encyclopedia of Philosophy (Held 2018) closely follows Redhead's analysis. ${ }^{6}$

\footnotetext{
${ }^{6}$ That this view is the most common does not mean that it is the only one. As we will see, Bell (1966) understood the theorem in a way that approaches the one I defend here.
} 


\subsection{HIDDEN VARIABLES AND THE FUNCTIONAL COMPOSITIONAL PRINCIPLE}

A HVT in Hilbert space can be naturally characterized by the following principles. Principle i) captures the spirit of HVTs in general, whereas ii) and iii) concern how a HVT in Hilbert space should represent its beables:

i) A quantum system has definite values for all its beables simultaneously, at all times.

ii) Given a Hermitian operator $O$, if there is an operationally defined real number $o$ that is probabilistically distributed according to the statistical algorithm of the quantum formalism for $O$-i.e., such that $\rho(o)=\left\langle\psi\left|P_{\varphi}\right| \psi\right\rangle$, or $\rho(o)=\operatorname{Tr} U P_{\varphi}$, where $P_{\varphi}$ is a projector in the spectral decomposition of $O$-then there exists a beable $\mathcal{O}$ associated with the number $O$ and that is measured by it. Thus, every Hermitian operator represents a beable.

iii) To every beable of a system, there corresponds one and only one Hermitian operator as its mathematical representative.

Consider now the following theorem in the quantum Hilbert space formalism. Take an arbitrary Hermitian operator $O$ representing observable $\mathcal{O}$, and let $\operatorname{Prob}\left[v(\mathcal{O})^{|\psi\rangle}=o\right]=\left\langle\psi\left|P_{\varphi}\right| \psi\right\rangle$ denote the probability that $v(\mathcal{O})^{|\psi\rangle}=o$, where $v(\mathcal{O})^{|\psi\rangle}$ is the value obtained in an $\mathcal{O}$-measurement on a system in an arbitrary state $|\psi\rangle$, and $P_{\varphi}$ is a projector onto $|\varphi\rangle$ in the spectrum of $O$, with eigenvalue $o$. It can be proven (see Redhead 1987, 18) that

$$
\operatorname{Prob}\left[v(f(\mathcal{O}))^{|\psi\rangle}=o\right]=\operatorname{Prob}\left[f\left(v(\mathcal{O})^{|\psi\rangle}\right)=o\right]
$$

where $f$ is an arbitrary function, and $f(\mathcal{O})$ is the observable whose Hermitian operator is $f(O)$. Let us call this theorem STAT FUNC (for reasons that will be clear shortly). STAT FUNC holds in the quantum formalism independently of considerations about hidden variables.

Let us now consider the functional compositional principle (FUNC), which states that in HVTs in Hilbert space the algebra of possessed values of beables mirrors the algebra of their representing Hermitian operators. That is, let $v(\mathcal{O})^{|\psi\rangle}$ now represent the value of a beable $\mathcal{O}$ possessed by a quantum system in an arbitrary state $|\psi\rangle$, and let $A$ and $B$ be two Hermitian operators representing beables $\mathcal{A}$ and $\mathcal{B}$, respectively, such that $B=f(A)$, where $f$ is an arbitrary function. FUNC states that

$$
v(\mathcal{B})^{|\psi\rangle}=f\left(v(\mathcal{A})^{|\psi\rangle}\right),
$$

or, alternatively, that

$$
v(f(\mathcal{A}))^{|\psi\rangle}=f\left(v(\mathcal{A})^{|\psi\rangle}\right)
$$

where $f(\mathcal{A})$ is the beable whose Hermitian operator is $f(A)$. 
It turns out that FUNC-which is not a theorem in the quantum formalism-follows from the conjunction of i), ii, iii) and STAT FUNC. Take an arbitrary state $|\psi\rangle$ and, given iii), a beable $\mathcal{A}$ represented by the operator $A$. By i), the system possesses a definite numerical value $v(\mathcal{A})^{|\psi\rangle}$ for $\mathcal{A}$. We can then form the number $f\left(v(\mathcal{A})^{|\psi\rangle}\right)$ with an arbitrary function $f$. By STAT FUNC,

$$
\operatorname{Prob}\left[f\left(v(\mathcal{A})^{|\psi\rangle}\right)=b\right]=\operatorname{Prob}\left[v(f(\mathcal{A}))^{|\psi\rangle}=b\right]
$$

which implies that the number $f\left(v(\mathcal{A})^{|\psi\rangle}\right)$ is probabilistically distributed by the statistical algorithm of the quantum formalism for the operator $f(A)$. Thus, by ii), there is a beable $\mathcal{B}$ represented by $B=$ $f(A)$, and measured by the number $f\left(v(\mathcal{A})^{|\psi\rangle}\right)$. In turn, by i), $|\psi\rangle$ has a definite value $v(\mathcal{B})^{|\psi\rangle}$ for $\mathcal{B}$. Finally, by ii) and iii), $\mathcal{B}$ is the only beable corresponding to $B=f(A)$, so that $v(\mathcal{B})^{|\psi\rangle}=f\left(v(\mathcal{A})^{|\psi\rangle}\right)$, which is FUNC. In short, although FUNC is not a theorem in the quantum formalism, it does hold in HVTs characterized by i), ii) and iii).

If we read STAT FUNC as the statistical version of FUNC, that is, as a statement about long-run measured frequencies of possessed beable values, we can derive it from FUNC. If the algebra of possessed values mirrors the algebra of the corresponding operators (FUNC), so must the long-run frequencies of the measured values (STAT FUNC). ${ }^{7}$ In other words, HVTs characterized by i), ii) and iii) offer a natural explanation of STAT FUNC.

Let now $Q$ be an arbitrary non-degenerate Hermitian operator in a Hilbert space $\mathcal{H}$ of dimension 3 or greater, with eigenvalues $q_{1}, q_{2}, q_{3}, \ldots$, and eigenvectors $\left|q_{1}\right\rangle,\left|q_{2}\right\rangle,\left|q_{3}\right\rangle, \ldots$. Then take the projectors $P_{1}, P_{2}, P_{3}, \ldots$ onto $\left|q_{1}\right\rangle,\left|q_{2}\right\rangle,\left|q_{3}\right\rangle, \ldots$, respectively. From FUNC and the properties of projectors, it can be proven (see Redhead 1987, 121-123) that

and that

$$
v\left(\mathcal{P}_{i}\right)^{|\psi\rangle}=1 \text { or } 0,
$$

$$
v\left(\mathcal{P}_{1}\right)^{|\psi\rangle}+v\left(\mathcal{P}_{2}\right)^{|\psi\rangle}+v\left(\mathcal{P}_{3}\right)^{|\psi\rangle}+\cdots=1
$$

Thus, an assignment of values for the beables $\mathcal{P}_{i}$ is constrained by FUNC in such a way that one of them must have value 1 , while the rest must have value 0 .

An arbitrary choice of several non-commuting $Q, Q^{\prime}, Q^{\prime \prime}, \ldots$ with corresponding orthogonal projectors $P_{i}, P_{i}^{\prime}, P_{i}^{\prime \prime}, \ldots$ determines a collection of sets of orthogonal rays in $\mathcal{H}$ (one set for the $P_{i}$, one set for the $P_{i}^{\prime}$, and so on). Thus, the constraint imposed by FUNC on the value assignments for the $\mathcal{P}_{i}, \mathcal{P}_{i}^{\prime}, \mathcal{P}_{i}^{\prime \prime}, \ldots$ amounts to an equivalent constraint on an assignment of numbers for the rays in each set.

Given i) and ii), the assignment must be complete: no ray can be left unassigned. That is, a FUNCconstrained complete assignment of values for our rays must be such that, in each and every set, one ray is assigned the number 1 while the rest are assigned the number 0 . Now, since the dimension of $\mathcal{H}$ is at least 3 , the chosen $Q, Q^{\prime}, Q^{\prime \prime}, \ldots$ may be such that some pairs of operators share an eigenvector, so the same ray may belong to more than one set. Given iii), the assignment must be consistent, in

\footnotetext{
${ }^{7}$ Assuming that measurements are faithful, in the sense that the possessed beable value just before the measurement is the same value that the measurement yields.
} 
the sense that each ray must be unambiguously assigned a single number in all the sets it belongs to, otherwise we would have a complete but contradictory assignment of beable values. ${ }^{8}$

In short, our main result so far is the following: a HVT characterized by i), ii) and iii) is necessarily committed to a complete and consistent FUNC-constrained assignment of possessed beable values.

\subsection{THE THEOREM}

The KST is a proof of the impossibility of a FUNC-constrained complete and consistent assignment for all the projectors, or the corresponding rays, in a Hilbert space of dimension 3 and higher. The proof proceeds by illustrating the impossibility of the assignment for a given collection of triads of orthogonal rays in $\mathcal{H}^{3}$. That is, given a certain collection of triads, the theorem shows that any attempt to implement a FUNC-constrained complete and consistent assignment leads to a contradiction. Kochen and Specker's (1967) proof involves 117 rays, but later proofs that are much more economic. Peres (1991) presents a very simple and elegant one using only 33 rays. ${ }^{9}$

A subtle point that will be crucial below is the following. Consider for a moment the KST no-go result from a strictly mathematical point of view. That is, given our collection of triads of orthogonal rays, it is impossible to assign in each triad the number 1 to one ray and the number 0 to the others in a complete and consistent way, whatever the physical meaning we assign to the rays may be. Now, we may interpret this mathematical fact, in physical terms, in a stronger or a in a weaker way. In the stronger reading - as in Redhead's analysis - the FUNC-constrained complete and consistent assignment of values is an assignment of possessed beable values, so we obviously assume that the Hermitian operators that determine the rays are representatives of beables. Thus, as an instance of the stronger reading, which is the usual way to understand the theorem in its relevance for HVTs, what Redhead shows is that a HVT in Hilbert space characterized by i), ii) and iii), is doomed to fall prey to the KST contradiction.

However, we could also take a weaker stand point and simply assume that the operators represent observables (see fn. 5). In this case, the impossible assignment is an assignment of (eigen)values as numerical outcomes of experiments. But then the KST must be stated in counterfactual terms. That is, if a measurement of observable $\mathcal{P}_{i}$ (whose operator $P_{i}=f Q=g Q^{\prime}$ projects onto a ray shared by the spectra of the non-commuting operators $Q$ and $Q^{\prime}$ ) yields an (eigen)value 1 when measured through $\mathcal{Q}$, then if it would have been measured through $\mathcal{Q}^{\prime}$ we would have obtained an (eigen)value 0 . In this weaker way to understand it, the KST imposes a constraint only on the (counterfactual) phenomenology of any quantum theory. So, if the KST is to have any ontological import on the beables in HVTs, these must be assumed to be represented by Hermitian operators, as in Redhead's stronger construal.

\footnotetext{
${ }^{8}$ It is sometimes stated that this consistency constraint amounts to a non-contextuality assumption (e.g., see Held 2018). I think the theorem is better presented if we refrain from using the "(non)contextuality" talk until the no-go result is derived. If non-contextuality lies on the consistency requirement, then the rejection of that requirement (the rejection of iii)) should lead to a form of contextuality. But, as we will see, the KST imposes a contextuality constraint also if either i) or ii) is rejected.

${ }_{9}^{9}$ Some approaches treat the KST vis-à-vis Bell's theorem, with the aim of addressing the connections between contextuality and non-locality. As Aravind (1999) shows, proofs of the KST can be turned into Bell's theorem proofs. The KS-Bell proofs, though, require entangled states, whereas the KST holds for arbitrary states. We are interested in the KST in the light of vNT and GT, which do not restrict their scope to entangled states either. Besides, a stateindependent approach is adequate for addressing the ontological import of the KST.
} 
It seems natural then to consider the strong interpretation in order to address the relevance of the KST for HVTs, which explains why an analysis like Redhead's has become the usual stance on the theorem. Let us thus take a closer look at the nature of the ontological contextuality for beables that follows from the strong reading.

\subsection{ONTOLOGICAL CONTEXTUALITY}

A HVT that observes i), ii), and iii) would not be affected by the measurement problem, and it would naturally explain the probabilistic nature of the predictions of the quantum formalism: like in statistical mechanics, the probabilities would be a matter of our ignorance. Besides, FUNC would be a logical consequence in it, so the theory would also provide a natural explanation of STAT FUNC. Hence the alleged relevance of the KST: a theory observing i), ii) and iii) yields a FUNCconstrained complete and consistent assignment of values for the beables of a system, but the KST shows that such an assignment is impossible.

To further illustrate, let $A, B$ and $C$ be Hermitian operators that share an eigenvector, and such that $C=f(A)=g(B)$ and $[A, B] \neq 0$, whereas $[C, A]=[C, B]=0 .{ }^{10}$ FUNC imposes that $v(\mathcal{C})^{|\psi\rangle}=$ $f\left(v(\mathcal{A})^{|\psi\rangle}\right)=g\left(v(\mathcal{B})^{|\psi\rangle}\right)$, but the KST shows that there is no complete and consistent assignment of values that observes this constraint. Thus, by modus tollens, the KST compels HVTs to drop one of the assumptions i), ii), iii) (recall that STAT FUNC is a theorem). Now, the rejection of any of the three assumptions implies some form of contextuality:

a) By dropping i) a system can have definite values at all times for a certain set of beables, but not for all of them. For beables like $\mathcal{C}$, the system possesses a definite value only relatively to a specific context of measurement, i.e., depending on whether we measure $\mathcal{C}$ via $\mathcal{A}$ or via $\mathcal{B}-$ so that it is possible that $f\left(v(\mathcal{A})^{|\psi\rangle}\right) \neq g\left(v(\mathcal{B})^{|\psi\rangle}\right)$.

b) We drop ii) by stating that even though the numbers $f\left(v(\mathcal{A})^{|\psi\rangle}\right)$ and $g\left(v(\mathcal{B})^{|\psi\rangle}\right)$ are both probabilistically distributed by the statistical algorithm of the quantum formalism for $f(A)$ and $g(B)$, respectively, $f(A)$ represents a beable, but $g(B)$ does not. This amounts to state that whether $C$ represents a beable or not depends on the measurement context: measuring beable $\mathcal{A}$ and applying $f$ yields a beable value, but measuring beable $\mathcal{B}$ and applying $g$ yields a physically meaningless number.

c) Dropping iii) by stating that $C$ represents two different beables $f(\mathcal{A})$ and $g(\mathcal{B})$ introduces contextuality insofar as which beable $C$ represents each time depends on the context of measurement.

\footnotetext{
${ }^{10}$ If a Hermitian operator $C$ is degenerate, it can be written as a function of an infinite number of different nondegenerate Hermitian operators, where all such operators that $C$ is a function of do not commute with each other, though $C$ commutes with each one of them (see Redhead 1987, 19-22). Intuitively, we only need to fix the shared eigenvector and rotate the triad about it to obtain infinitely many triads corresponding to infinitely many operators that do not commute with $A$, but that do commute with $C$.
} 
All three options have radical consequences for the ontology of beables. Strategy a) involves a compromise in the spirit of HVTs. The gist of these theories assumes that there is an external determinate quantum reality in which physical systems have definite values for all its properties at all times, regardless of whether measurements are performed or not. However, the rejection of i) implies a Bohrian ontological description of the contextual beables: they cannot have definite values independently of measurements. HVTs aim to remove fuzziness or indeterminacy for beable values, but for contextual beables like $\mathcal{C}$, fuzziness and indeterminacy come up anyway.

Strategy b) leaves us in a scenario in which even though $C=f(A)=g(B)$, where $A, B$ and $C$ represent the beables $\mathcal{A}, \mathcal{B}$ and $\mathcal{C}$, respectively, either $f(A)$ or $g(B)$ does not represent a beable. Now, the idea that even though $\mathcal{A}$ and $\mathcal{B}$ are both beables, if we apply a function on one of the representative operators we define a new beable, whereas if we do the same with the other operator no beable gets defined, is hard to justify. It is difficult to conceive a physically plausible criterion to ground this disparity.

Finally, strategy c) involves an infinite multiplication of beables for operators like $C$ (see $\mathrm{fn}$. 10). Apart from this seemingly ad-hoc de-Ockhamization of properties, further ontological questions ensue. For example, let us take again the operator $C=f(A)=g(B)$ which in this view represents (at least) two different beables. In an $\mathcal{A}$-measurement, the value of $f(\mathcal{A})$ depends on the value of the beable $\mathcal{A}$. But what about the value of $f(\mathcal{A})$ in a $\mathcal{B}$-measurement context? Is the beable $f(\mathcal{A})$ still an element of reality in that case? If it is, does its value depend on $v(\mathcal{A})$ ? Can we alter the value of $f(\mathcal{A})$, or turn off the beable itself from existence, just by switching between an $\mathcal{A}$-measurement context and a $\mathcal{B}$-measurement context?

As I mentioned above, Redhead's analysis of the structure and premises of the theorem is very useful here. By showing that the conjunction of STAT FUNC, i), ii), and iii) leads to the KST contradiction, it allows us to precisely spell out the types of contextuality that the theorem allegedly imposes on HVTs in Hilbert space. Most importantly, when contextuality is described accurately in the discussion of the theorem, we see that in all three forms it concerns the ontology of beables in HVTs.

Alternatives b) and c) are rarely defended in the literature, though, and the KST is usually taken to compel HVTs to choose a). Bub (1997), for example, characterized the challenge of a realist interpretation of quantum mechanics as finding out what is the maximum number of beables that can have objective definite values without falling prey to the KST (notice that Bub's is yet another example of the usual interpretation of the KST). However, Redhead's analysis is useful in clarifying that i) can be retained, but on the price of some other bizarre forms of ontological contextuality.

Summarizing so far, a HVT characterized by i), ii) and iii) is necessarily committed to a FUNCconstrained complete and consistent assignment of beables values. The KST shows that such an assignment is impossible, so it forces HVTs in Hilbert space to drop either i), ii), or iii). In turn, this leads to some form of ontological contextuality for the beables in the theory, in the form of a), b), or c), respectively.

\section{VON NEUMANN'S THEOREM}

In 1932, in his seminal Mathematical Foundations of Quantum Mechanics, John von Neumann (1955) derived a theorem that was understood by many as a proof of the impossibility of such theories (but 
see fn. 3). John Bell (1966) contested this reading, and claimed that the only relevance of vNT consists in that it rules out an uninteresting class of HVTs. However, Jammer (1974) and Bub $(2010,2011)$ have shown that vNT does convey an interesting result. Jammer states that the theorem proves that quantum mechanics cannot be embedded in a HVT within Hilbert space, and Bub states that the theorem establishes that in HVTs the beables of quantum systems cannot be represented by Hermitian operators. vNT is mostly known through Bell's (1966) article, that does not present the proof in detail. However, an accurate understanding of its true meaning requires a more detailed scrutiny.

\subsection{THE PROOF}

In 1932, von Neumann (1955) published his seminal Mathematical Foundations of Quantum Mechanics. There he showed that the quantum theory that Heisenberg, Jordan \& Born (1926) and Schrödinger (1926) had articulated in the form of matrix and wave mechanics, respectively, could be solidly and elegantly formulated in the mathematical framework that we nowadays call Hilbert space. In section III.1 of his book, he showed that a generalized expression for the Born rule could be formulated in Hilbert space in the following way, where $U$ is a density operator, and $R$ is a Hermitian operator representing observable $\mathcal{R}$ :

$$
\langle\mathcal{R}\rangle_{U}=\operatorname{Tr}(U R)
$$

Given the probabilistic character of (1), in chapter IV von Neumann ponders if it could be interpreted in a classical way, that is, as a matter of our ignorance. To treat this issue he introduces two definitions. First, a state $U$ is dispersion-free iff there is no statistical spread in the predictions of measurement outcomes, i.e., such that for every $\mathcal{R}$,

$$
\langle\mathcal{R}\rangle_{U}^{2}=\left\langle\mathcal{R}^{2}\right\rangle_{U}
$$

Second, a system is in a homogeneous state $U$ iff for any subensembles $U_{1}$ and $U_{2}$,

$$
\langle\mathcal{R}\rangle_{U}=\langle\mathcal{R}\rangle_{U_{1}}=\langle\mathcal{R}\rangle_{U_{2}}
$$

By definition, in a HVT that provides an ignorance interpretation of (1), there must be dispersionfree states. Furthermore, since from a HVT perspective a dispersive state is not completely described, such a system could be split into dispersion-free subsystems, according to suitable values of the hidden variables. Thus, in a HVT, a dispersive state cannot be homogeneous.

Von Neumann then introduces four "general qualitative assumptions" $(1955,295)$ :

$\mathbf{A}^{\prime}$. If observable $\mathcal{R}$ is by nature a non-negative quantity, then $\langle\mathcal{R}\rangle \geq 0$.

$\mathbf{B}^{\prime}$. If $\mathcal{R}, \mathcal{S}, \ldots$ are arbitrary observables and $a, b, \ldots$ are real numbers, then $\langle a \mathcal{R}+b \mathcal{S}+\cdots\rangle=a\langle\mathcal{R}\rangle+$ $b\langle\delta\rangle+\cdots$.

I. If the observable $\mathcal{R}$ is represented by the Hermitian operator $R$, then the observable $f(\mathcal{R})$ is represented by the Hermitian operator $f(R)$. 
II. If observables $\mathcal{R}$ and $\mathcal{S}$ are represented by the Hermitian operators $R$ and $S$, respectively, the observable $\mathcal{R}+S$ is represented by the Hermitian operator $R+S$, regardless of whether $R$ and $S$ commute or not.

From these four assumptions, von Neumann (1955, 313-317) derives (1), and then he shows that it does not allow dispersion-free states, whereas it does admit homogeneous ones.

The proof that there are no dispersion-free states is simple (von Neumann 1955, 320-321). Recall that a deterministic state $U$ is such that $[\operatorname{Tr}(U R)]^{2}=\operatorname{Tr}\left(U R^{2}\right)$. Choosing for $R$ a projector $P_{\varphi}$ onto an arbitrary normalized vector $|\varphi\rangle$, the idempotency of $P_{\varphi}$ implies that $\left[\operatorname{Tr}\left(U P_{\varphi}\right)\right]^{2}=\operatorname{Tr}\left(U P_{\varphi}\right)$, so that $\left\langle P_{\varphi}|U| P_{\varphi}\right\rangle^{2}=\left\langle P_{\varphi}|U| P_{\varphi}\right\rangle$, and then $\left\langle P_{\varphi}|U| P_{\varphi}\right\rangle$ must equal 1 or 0 . Let us now take two normalized vectors $\left|\varphi^{\prime}\right\rangle$ and $\left|\varphi^{\prime \prime}\right\rangle$, and vary continuously from one to the other (von Neumann proved that all vectors $|\varphi\rangle$ along the variation are normalized). Since the vector variation is continuous, the variation from $\left\langle P_{\varphi \prime}|U| P_{\varphi \prime}\right\rangle$ to $\left\langle P_{\varphi \prime \prime}|U| P_{\varphi \prime \prime}\right\rangle$ must also be continuous. Thus, given that $\left\langle P_{\varphi}|U| P_{\varphi}\right\rangle$ has to be either 1 or $0,\left\langle P_{\varphi}|U| P_{\varphi}\right\rangle$ must be constant along the variation, and $\left\langle P_{\varphi \prime}|U| P_{\varphi \prime}\right\rangle=\left\langle P_{\varphi \prime \prime}|U| P_{\varphi \prime \prime}\right\rangle$. Thus, along the whole variation either $U=I$ or $U=0$. But $\operatorname{Tr} U=1$, so no $U$ can be dispersion-free.

The proof that there are homogeneous states (von Neumann 1955, 321-322) is somewhat more involved. Let $U_{1}$ and $U_{2}$ be any subensembles of $U$, and assume $U$ is homogeneous. With $c^{\prime}>0$, $c^{\prime \prime}>0$, and $c^{\prime}+c^{\prime \prime}=1$, it follows from the homogeneity condition above that $U=c^{\prime} U_{1}+c^{\prime \prime} U_{2}$ implies that $U_{1}=c^{\prime} U$ and $U_{2}=c^{\prime \prime} U$. Let $\left|\varphi_{+}\right\rangle$be a vector such that $U\left|\varphi_{+}\right\rangle \neq 0$, and define the operators

$$
V|\varphi\rangle=\frac{\left\langle\varphi|U| \varphi_{+}\right\rangle}{\left\langle\varphi_{+}|U| \varphi_{+}\right\rangle} \cdot U\left|\varphi_{+}\right\rangle, \quad W|\varphi\rangle=U|\varphi\rangle-V|\varphi\rangle
$$

where $|\varphi\rangle$ is any vector. Von Neumann proved that $V$ and $W$ are positive definite. Thus, given that $U=V+W$, if $U$ is homogeneous, then $V=c^{\prime} U$; and since $V\left|\varphi_{+}\right\rangle=U\left|\varphi_{+}\right\rangle, c^{\prime}=1$, so that $V=U$. Von Neumann then defined the normalized vector $|\psi\rangle=\frac{U\left|\varphi_{+}\right\rangle}{\| U\left|\varphi_{+}\right\rangle \|}$and the constant $c=\frac{\| U\left|\varphi_{+}\right\rangle \|^{2}}{\left\langle\varphi_{+}|U| \varphi_{+}\right\rangle}$to obtain

$$
U|\varphi\rangle=V|\varphi\rangle=c|\psi\rangle\langle\psi \mid \varphi\rangle=c P_{\psi}|\varphi\rangle
$$

so that $U=P_{\psi}$. That is, if $U$ is homogenous, it is a projector.

Conversely, assume that $U=P_{\psi}$, where $|\psi\rangle$ is normalized, and that $U=V+W$, where $V$ and $W$ are positive definite. Then for any vector $|\varphi\rangle, 0 \leq\langle\varphi|V| \varphi\rangle \leq\langle\varphi|V| \varphi\rangle+\langle\varphi|W| \varphi\rangle=\langle\varphi|U| \varphi\rangle$. But if $U|\varphi\rangle=0$, then $\langle\varphi|U| \varphi\rangle=0$, so that $\langle\varphi|V| \varphi\rangle=0$, and $V|\varphi\rangle=0$. That is, if $U|\varphi\rangle=0$, then $V|\varphi\rangle=0$. Now, for any vector $|\varphi\rangle$ orthogonal to $|\psi\rangle, U|\varphi\rangle=0$, for if $\langle\varphi \mid \psi\rangle=0$, then $U|\varphi\rangle=P_{\psi}|\varphi\rangle=0$, and $V|\varphi\rangle=0$. Now, since for any vector $|\theta\rangle$ it holds that $\langle\theta|V| \varphi\rangle=\langle\varphi|V| \theta\rangle$, any vector $|\varphi\rangle$ that is orthogonal to $|\psi\rangle$ (so that $V|\varphi\rangle=0$ ) is orthogonal to $V|\theta\rangle$ as well. Hence, $V|\theta\rangle=c_{\theta}|\psi\rangle$, where $c$ depends on $|\theta\rangle$. So if $|\theta\rangle=|\psi\rangle$, then $V|\psi\rangle=c^{\prime}|\psi\rangle$. Now, any vector $|\varphi\rangle$ can be written in the form $\langle\psi \mid \varphi\rangle|\psi\rangle+\left|\varphi^{\prime}\right\rangle$, where $\left|\varphi^{\prime}\right\rangle$ is orthogonal to $|\psi\rangle$. Therefore,

$$
V|\varphi\rangle=\langle\psi \mid \varphi\rangle \cdot V|\psi\rangle+V\left|\varphi^{\prime}\right\rangle=c^{\prime}|\psi\rangle\langle\psi \mid \varphi\rangle=c^{\prime} P_{\psi}|\varphi\rangle=c^{\prime} U|\varphi\rangle
$$

and finally, $V=c^{\prime} U$ and $W=U-V=\left(1-c^{\prime}\right) U$. That is, if $U$ is a projector, it is homogeneous. 
We have then a proof that $U$ is homogeneous iff it is a projector. Thus, the quantum states allowed by (1) are always dispersive, and some of them are homogeneous. It follows that under assumptions $\mathbf{A}^{\prime}, \mathbf{B}^{\prime}, \mathbf{I}$ and II, HVTs are not possible.

Notice that this result involves two main steps. First, a derivation of (1) using $\mathbf{A}^{\prime}, \mathbf{B}^{\prime}, \mathbf{I}$ and II; and then a proof that (1) does not allow dispersion-free states but allows homogeneous states, in which premise $\mathbf{B}^{\prime}$ is not used-although the result of the second step is conditional on the premises in the first step, of course. Most accounts of vNT follow Bell's (1966) exposition, but the reader familiar with it will notice that the present review, which follows von Neumann quite closely, is significantly different from Bell's presentation. As we will see, a faithful display of von Neumann's two steps is crucial in order to properly understand the significance of the theorem, and to understand why GT is another proof of the same result on the basis of weaker assumptions.

\subsection{BELL'S CRITIQUE}

Bell claims that the main assumption in vNT is that "any linear combination of any two Hermitian operators represents an observable, and the same combination of expectation values is the expectation value of the combination" $(1966,448)$, that is, II and B'. He then asserts that this assumption "is required by von Neumann of the hypothetical dispersion-free states also". Now, "for a dispersionfree state (which has no statistical character) the expectation value of an observable must equal one of its eigenvalues" $(1966,449)$. But if an observable $\mathcal{O}$ is represented by $O=R+S$, where $[R, S] \neq 0$, the eigenvalues of $O$ are, in general, not equal to a sum of the eigenvalues of $R$ and $S$. According to Bell, von Neumann then concludes that dispersion-free states - and hence HVTs - are tout court impossible.

The core of Bell's criticism of vNT is his rejection of $\mathbf{B}^{\prime}$ for dispersion-free states:

The essential assumption can be criticized as follows. At first sight the required additivity of expectation values seems very reasonable, and it is rather the nonadditivity of allowed values (eigenvalues) which requires explanation. Of course the explanation is well known: a measurement of a sum of noncommuting observables cannot be made by combining trivially the results of separate observations of the two terms-it requires a quite different experiment. (Bell 1966, 449)

As an example, Bell refers to the operator $\sigma_{x}+\sigma_{y}$, representing the spin observable $\delta_{x}+\delta_{y}$. The eigenvalues of $\sigma_{x}$ and $\sigma_{y}$ are \pm 1 , but the eigenvalues of $\sigma_{x}+\sigma_{y}$ are $\pm \sqrt{2}$, so von Neumann's (alleged) constraint cannot be observed. However, since $\left[\sigma_{x}, \sigma_{y}\right] \neq 0, \delta_{x}+\mathcal{S}_{y}$ cannot be measured by adding the values obtained in separate measurements of $\delta_{x}$ and $\delta_{y}$. Thus, the non-additivity of the expected values of $\mathcal{S}_{x}+\mathcal{S}_{y}$ for dispersion-free states is natural, despite the additivity of the statistical expectation values.

Bell then states that the theorem does not support von Neumann's (supposed) conclusion that HVTs with dispersion-free states are impossible:

It was not the objective measurable predictions of quantum mechanics which ruled out hidden variables. It was the arbitrary assumption of a particular (and impossible) relation between the results of incompatible measurements either of which might be made on a given occasion but only one of which can in fact be made. $(1966,449)$ 
Thus, Bell shows that vNT does not prove that HVTs are impossible. His criticism goes further, though: since the imposition of $\mathbf{B}^{\prime}$ ' on dispersion-free states is, he claims, unjustified, the theorem does not have much importance: it only rules out the uninteresting class of HVTs that observe $\mathbf{B}^{\prime}$. This has become the general opinion in the philosophy and foundations of physics community.

\subsection{A REASSESSMENT}

Bell complains that for incompatible measurements, additivity of expectation values for deterministic states, i.e., $\mathbf{B}^{\prime}$, is an unjustified assumption. However, von Neumann does offer arguments for the four assumptions, including $\mathbf{B}^{\prime}$. $\mathbf{A}^{\prime}$ is practically analytic, and required to prove that in (1) $U$ is positive-definite (von Neumann 1955, 317). The justification for I and II corresponds to the representation of quantities by Hermitian operators. Von Neumann explicitly remarks that "in quantum mechanics [...], the quantities $\mathcal{R}$ correspond one-to-one to the hypermaximal Hermitian operators $R^{\prime \prime}(1955,247)$. Then, commenting on II, he adds that "this operation depends on the fact that for two Hermitian operators, $R, S$, the sum $R+S$ is also a Hermitian operator, even if the $R, S$ do not commute" (1955, 309). I and II thus guarantee that the functional relations between the represented quantities (beables, if we discuss HVTs) are mirrored by the representing operators.

Regarding B', von Neumann introduces it on the grounds of a general principle in physical reasoning, and in order to guarantee the functional mirroring established by I and II for beables of the form $\mathcal{R}+\mathcal{S}$, when $[R, S] \neq 0$. Just before addressing the subject of expectation values as a preliminary for the theorem, von Neumann asks the reader to "forget the whole of quantum mechanics", but retaining the following basic principle $(1955,297)$. If we measure a quantity $\mathfrak{R}$ on a system $\boldsymbol{S}$ and obtain a value $a$, then we can always take a function $\mathbf{f}(x)$, define the quantity $\mathbf{f}(\mathfrak{R})$, and obtain the value $\mathbf{f}(a)$ (this is the general foundation for $\mathbf{I}$, of course).

But assume now (still independently of quantum theory) that the quantities $\Re$ and $\subseteq$ are experimentally incompatible. It seems problematic to even define a quantity $\mathbf{f}(\mathfrak{R}, \mathfrak{S})$, for there is no possible experimental setup that can measure $\mathfrak{R}$ and $\subseteq$ simultaneously, nor successively on a system in the same state. However, von Neumann $(1955,298-300)$ refers to an ensemble of systems $\boldsymbol{S}_{1}$, $\boldsymbol{S}_{2}, \ldots, \boldsymbol{S}_{N}$, with $N$ large. In measurements on this ensemble, we do not get the value of a quantity, but a distribution of values. Now, even if two quantities $\Re$ and $\subseteq$ are incompatible, we can take different subsets of the ensemble, with $M$ elements (where $M \ll N$, but $M$ large), and separately measure $\Re$ and $\subseteq$ in these sub-ensembles. These measurements yield the statistical distribution of both $\Re$ and $\subseteq$ for the whole ensemble, and with these distributions we are now able to define our quantity $\mathbf{f}(\mathfrak{R}, \mathbb{S})$ anyway. In order to do this, we must assume that $\boldsymbol{B}^{\prime}$ holds for the expectation values of ensembles. Thus, von Neumann's justification for this assumption is that it is required to functionally define quantities that are a linear combination of experimentally incompatible observables. In other words (and now returning to quantum theory), in order to extract the full representational power of Hilbert space according to I and II, i.e., respecting the principle that the functional relations between quantities are mirrored by the representing operators, $\mathbf{B}^{\prime}$ is required. Thus, $\mathbf{B}^{\prime}$, more than a physical assumption, is a principle that allows the definition of quantities like $\mathbf{f}(\mathfrak{R}, \subseteq)$. 
Although von Neumann is not explicit about it, a very important point to notice here is that this rationale for $\mathbf{B}^{\prime}$ does not require that the expectation values must be probabilistically spread. Von Neumann's argument simply allows us to deal with the experimental incompatibility between the quantities $\mathfrak{R}$ and $\subseteq$ that seems to jeopardize the definition of the quantity $\mathbf{f}(\mathfrak{R}, \subseteq)$ - recall that he asks us to forget quantum mechanics for a second. Actually, this reasoning to deal with experimentally incompatible quantities would also be applicable in a context in which the measurements on the sub-ensembles yield deterministic outcomes. It is not the probabilistic nature of quantum mechanics that complicates the possibility of functionally defining $\mathbf{f}(\mathfrak{R}, \subseteq)$, but the experimental incompatibility between $\mathfrak{R}$ and $\subseteq$. Consequently, there are no reasons to restrain the scope of $\mathbf{B}^{\prime}$ to dispersive states. Actually, since von Neumann is pondering about the possibility of deterministic states in quantum mechanics, it is quite natural that he does not do it.

Von Neumann (1955, 309, fn. 164) offers an example of his justification of $\mathbf{B}^{\prime}$ referring to the energy operator $H$, which is a linear combination of the non-commuting position and momentum operators, that is $H=f(Q)+g(P)$. Although the energy quantity $\mathcal{E}$ cannot be measured by measuring simultaneously or successively the incompatible quantities position $\mathcal{Q}$ and momentum $\mathcal{P}, \mathcal{E}$ can still be defined as a function of $\mathcal{Q}$ and $\mathcal{P}$ through their expectation values, i.e., $\langle\mathcal{E}\rangle=f\langle\mathcal{Q}\rangle+g\langle\mathcal{P}\rangle$. In other words, without $\boldsymbol{B}^{\prime}$ we could not define energy as $\mathcal{E}=\mathbf{f}(\mathcal{Q}, \mathcal{P})$ - and, again, this reasoning does not need to assume that the expectation values have to be dispersive.

In short, von Neumann's reasoning is that I and II must be taken on board if we are going to use Hilbert space in its full representative power, following the general principle in physical reasoning that he asks us to retain. But given the experimental incompatibility between some quantities - those represented by non-commuting operators $-\mathbf{B}^{\prime}$ is needed to guarantee that representative power. That is, although $\mathbf{B}^{\prime}$ and II are logically independent, given that Hermitian operators in Hilbert space do not all commute, they are essentially linked in physical terms.

After our analysis of von Neumann's justification for $\mathbf{B}^{\prime}$, we can formulate the true meaning and relevance of his theorem. In 1935, Grete Hermann (2016, section 7) introduced a criticism that is similar in spirit to Bell's, and Max Jammer (1974, 272-275) offered a critical evaluation of Hermann's argument that clarifies the true significance of vNT. Hermann claimed that the proof is circular. Recall that $\mathbf{B}^{\prime}$ states that $\langle\mathcal{R}+\mathcal{S}\rangle=\langle\mathcal{R}\rangle+\langle\mathcal{S}\rangle$, even if $[R, S] \neq 0$. She points out that when $[R, S] \neq 0$, the expectation values of putative dispersion-free states for the beable $\mathcal{R}+\mathcal{S}$ may not be additive, given the value of the hidden variables. But then, when the dispersion-free states get averaged over values of the hidden variables in an ensemble, the expectation value may be indeed additive. Thus, Hermann claims, vNT begs the question insofar as $\mathbf{B}^{\prime}$ precludes the possibility of dispersion-free states: if $R$ and $S$ do not commute, deterministic states cannot observe $\mathbf{B}^{\prime}$.

Jammer states that although Hermann's criticism - just like Bell's - correctly points out that vNT is not a proof of the absolute impossibility of HVTs, her charge that the proof is circular is wrong, for $\mathbf{B}^{\prime}$ is not imposed on incompatible quantities and non-commuting operators only. That is, dispersion-free states for compatible quantities represented by commuting operators are logically consistent with $\mathbf{B}^{\prime}$, so the theorem is not question-begging. Now, although Jammer rightly rejects the circularity claim, he states that Hermann's argument allows us to properly understand the scope of vNT. By calling complete a theory that cannot be embedded in a HVT, he affirms that the insight in 
Hermann's criticism is that "von Neumann has proved that quantum mechanics is a complete theory, but only as far as quantum mechanical states are concerned" (1974, 273; my emphasis). He elaborates on this point in the following way:

We agree with Grete Hermann's criticism that the proof did not achieve its declared objective of demonstrating that quantum mechanical ensembles cannot be decomposed into any kind of dispersion-free subensembles [...]. But we do not dismiss the proof as nugatory. True, in view of von Neumann's excessively restricted assumptions it is not an impossibility proof of any conceivable class of hidden variables, but it is a completeness proof, in this respect, of von Neumann's axiomatics (with the inclusion of postulate [ $\left.\mathbf{B}^{\prime}\right]$ ), since it shows that this formalism does not admit nonquantum mechanical [dispersion-free] ensembles. (Jammer 1974, 274, fn. 45)

Given Jammer's definition of "complete", it is clear that he is not taking sides with Bohr in the Einstein-Bohr debate. He actually states that Hermann's argument shows that vNT is not a proof of the completeness, in Einstein's sense of "complete", of quantum mechanics, for HVTs are still possible. Rather, he argues, what the theorem really shows is that the standard quantum formalism (von Neumann's axiomatization) is complete in the sense that it cannot be embedded in a HVT in Hilbert space. In short, and using "complete" in a more comprehensive sense, Jammer's point is that vNT is a proof that quantum mechanics cannot be completed by a HVT within Hilbert space.

More recently, Jeffrey Bub $(2010 ; 2011)$ presented a reassessment of vNT along the same lines as Jammer. Paying close attention to the logical structure of the theorem, that is, that by assuming $\mathbf{A}^{\prime}$, B', I and II dispersion-free states are not possible, we see its real significance. Since, for HVTs, B', I and II amount to the principle that beables are represented by Hermitian operators in Hilbert space, he concludes that vNT is a proof that in viable HVTs such operators cannot be the representatives of the beables that configure their ontology:

what von Neumann's proof precludes, then, is the class of hidden variable theories in which i) dispersion-free (deterministic) states are the extremal states, and ii) the beables of the hidden variable theory correspond to the physical quantities represented by Hermitian operators of quantum mechanics. (2010, 1340; my emphasis)

After Bub's clarification, we can properly understand von Neumann's assessment of the importance of his proof in connection to the possibility of HVTs. He focuses on I and II as the assumptions that HVTs which admit dispersion-free states should violate:

We have even ascertained that it is impossible that the same physical quantities exist with the same function connections (i.e., that I, II hold), if other variables (i.e., "hidden parameters") should exist in addition to the wavefunction. (von Neumann 1955, 324-325) ${ }^{11}$

\footnotetext{
${ }^{11}$ In this passage von Neumann does not claim that HVTs are tout court impossible. However, a few lines after he writes "it is therefore not, as is often assumed, a question of a re-interpretation of quantum mechanics - the present system of quantum mechanics would have to be objectively false, in order that another description of the elementary processes than the statistical one be possible" $(1955,325)$. Whether von Neumann understood his theorem (wrongly) as an impossibility proof, or (rightly) as a constraint on HVTs, is a controversial issue - see Dieks (2017) for an opinionated treatment.
} 
Bell, though, claimed that $\mathbf{B}^{\prime}$ is the assumption to blame, so let us see what happens in a generic Hilbert space HVT that drops B' for deterministic states. Let $\mathcal{R}$ and $\mathcal{S}$ be two experimentally incompatible beables, represented by Hermitian operators $R$ and $S$, respectively. For dispersion-free states, the expectation values $\langle\mathcal{R}\rangle$ and $\langle\mathcal{S}\rangle$ are given by eigenvalues of $R$ and $S$, respectively. Now, for beable $Q$, represented by the operator $Q=R+S$, it holds by the same token that $\langle Q\rangle$, in the deterministic case, corresponds to an eigenvalue of $Q$, but since $[R, S] \neq 0$, the eigenvalues of $Q$ are not sums of eigenvalues of $R$ and $S$.

But then how could we state that $\mathcal{Q}=\mathcal{R}+\mathcal{S}$ ? We cannot simultaneously measure $\mathcal{R}$ and $\mathcal{S}$, nor can we measure them successively and then add the outcomes obtained to get the value of $\mathcal{Q}$. Furthermore, since we are assuming that $\mathbf{B}^{\prime}$ does not hold at the deterministic level for incompatible beables, we cannot invoke von Neumann's rationale to go around the experimental incompatibility and functionally define $\mathcal{Q}$-i.e, to define $\mathcal{Q}$ as $\mathbf{f}(\mathcal{R}, \mathcal{S})$-in terms of dispersion-free expectation values of different sub-ensembles. That is, at the deterministic level the expectation value of $Q$ is not a function of the expectation values of $\mathcal{R}$ and $\mathcal{S}$-i.e., $\langle\mathcal{Q}\rangle \neq\langle\mathcal{R}\rangle+\langle\mathcal{S}\rangle$. Thus, we conclude that the beable $\mathcal{Q}$ is not the beable that is the linear combination $\mathcal{R}+\mathcal{S}$, for $\mathcal{Q}$ cannot be functionally defined in terms of $\mathcal{R}$ and $\delta$. That is, without $B^{\prime}$, in a HVT in which $R, S$, and $Q$, each represents a beable, $Q$ is not a function $\mathbf{f}$ of $\mathcal{R}$ and $\mathcal{S}$-in short, $Q \neq \mathcal{R}+\mathcal{S}$ despite that $Q=R+S$. Consequently, the beable $\mathcal{R}+\mathcal{S}$ is not represented by the operator $R+S$, and it cannot even be defined functionally in terms of the mathematical representatives of $\mathcal{R}$ and $\mathcal{S}$. Thus, if $\mathbf{B}^{\prime}$ is dropped for dispersion-free states, so is II.

We could still take the experimental procedure associated to the operator $Q$, and define its outcome as the value of the beable we name " $\mathcal{R}+\mathcal{S}^{\prime}$, but that does not mean that the defined beable is a function of $\mathcal{R}$ and $\mathcal{S}$, of course. Let us illustrate with the Hamiltonian operator $H=f(Q)+g(P)$. We could stipulate in our putative HVT in Hilbert space that a usual $H$-measurement is a measurement of beable $\mathcal{E}$. However, given the incompatibility of the beables $\mathcal{Q}$ and $\mathcal{P}$, we cannot measure them jointly nor successively to obtain the value of $\mathcal{E}$. By assumption, $\mathbf{B}^{\prime}$ does not hold for the dispersion-free case, so we cannot use the additivity of expectation values to define $\mathcal{E}$ in functional terms of $\mathcal{Q}$ and $\mathcal{P}$ as $\langle\mathcal{E}\rangle=f\langle\mathcal{Q}\rangle+g\langle\mathcal{P}\rangle$. That is, although we could take a certain measurement outcome as the value of beable $\mathcal{E}$, call this beable "energy", and represent it by $H$, we cannot consider $\mathcal{E}$ as the beable that is the function $\mathbf{f}(\mathcal{Q}, \mathcal{P})=f(\mathcal{Q})+g(\mathcal{P})$. Without $\mathbf{B}^{\prime}$, a HVT in Hilbert space simply does not have the resources to define the beable $\mathbf{f}(Q, \mathcal{P})$ - the suitable operator $H$ is doing a different job. Furthermore, that additivity does hold at the dispersive level will not solve the problem - recall that the obstacle von Neumann identifies for the functional definition is experimental incompatibility, not the probabilities. Actually, in our putative HVT, additivity for dispersive states would be a feature loudly crying for an explanation, precisely because it does not hold at the level. ${ }^{12}$

The upshot is that if we jettison $\mathbf{B}^{\prime}$ for the deterministic case and we stay within the boundaries of Hilbert space, we cannot define the beable $\mathcal{R}+\mathcal{S}$ functionally in terms of $\mathcal{R}$ and $\mathcal{S}$ when $\mathcal{R}$ and $\mathcal{S}$ are experimentally incompatible. Thus, if $\mathbf{B}^{\prime}$ is violated at the dispersion-free level, so is II, so Bub's

\footnotetext{
${ }^{12}$ Empirical adequacy enforces that the trace rule (1) holds in a putative deterministic HVT for dispersive states. Thus, there must be a story in such a theory to justify (1) and to explain dispersive additivity. In Bohm's theory, that story is given by the dynamics of measurements and the role of Hermitian operators, which is not to represent beables (see section 5.2 below).
} 
evaluation of the theorem is correct. I also agree with Dieks' $(2017,141)$ appraisal (which is quite close to Jammer's) that "von Neumann has proved that viable hidden-variables theories cannot be Hilbert space theories". In other words, if we want to exploit the full representational power of Hermitian operators in Hilbert space with respect to beables, respecting the principle that the functional relations between quantities are mirrored by the representing operators, we certainly need $\mathbf{B}^{\prime}$. But then dispersion-free states are not possible. If we want them to be possible, we must reject $\mathbf{B}^{\prime}$ for dispersion-free states (as Bell rightly noted), but then II cannot hold either, so Hilbert space is not enough to represent the theory's beables. ${ }^{13}$

Summing up, Bell is correct in that in a putative HVT that observes B' and II, the expectation values for operators which are a linear combination of non-commuting operators should be additive in the hypothetical dispersion-free case, which leads to a contradiction. But this conclusion runs short. The truly important point is that if von Neumann's four assumptions hold, there are no dispersion-free states. Von Neumann does not arbitrarily impose $\mathbf{B}^{\prime}$ on deterministic states, he uses it as a principle that allows us to functionally define observables like $\mathcal{R}+S$ in Hilbert space, and then it turns out that deterministic states are not allowed by (1). Bell's analysis was very important in clarifying that vNT is not a general impossibility proof, but his disdainful evaluation misses the highly significant result that Hilbert space HVTs are not possible.

Anyhow, Bell's criticism prompts a very interesting question: can the exact same result of vNT be obtained from weaker assumptions that do not entail the additivity of dispersion-free expectation values for non-commuting operators?

\section{VON NEUMANN AND GLEASON}

The answer to this question is affirmative. Andrew Gleason (1957) obtained the result that in a Hilbert space $\mathcal{H}$ of dimension 3 and higher, every measure $\mu$ on a subspace $A$ of $\mathcal{H}$ is given by (1), that is, $\mu(A)=\operatorname{Tr}\left(U P_{A}\right)$, where $P_{A}$ is the projector onto $A$, and $U$ is a positive-definite, trace-one operator. The crucial premises in GT are that $\langle I\rangle=1$, and that for mutually orthogonal projectors such that $\sum P_{i}=I$, it holds that $\left\langle\sum P_{i}\right\rangle=\sum\left\langle P_{i}\right\rangle$.

Between vNT and GT there is indeed a very close connection. Both theorems show that if all possible measurement outcomes are represented by projection operators acting in a Hilbert space $\mathcal{H}$, the algorithm to assign probability measures on those outcomes is given by the trace rule $\mu(A)=$ $\operatorname{Tr}\left(U P_{A}\right)$. Thus, the quantum states admitted by this algorithm are exclusively given by the density

\footnotetext{
${ }^{13}$ In a reply to Bub (2010) and Dieks (2017), Mermin \& Schack (2018) defend the Hermann-Bell objection by claiming that even if $\mathbf{B}^{\prime}$ is rejected for incompatible quantities in the dispersion-free case, II still allows to define quantities like $\mathcal{R}+\mathcal{S}$ when $[R, S] \neq 0$. In their own words, "assumption II provides a powerful alternative way to define linear combinations of physical quantities that are not jointly measurable. In terms of that definition it is not only meaningful to reject $\mathbf{B}$ ' for the hypothetical dispersion-free subensembles, but quite compatible with the general structure of ordinary quantum mechanics" (Mermin \& Schack 2018, 1013; my italics). After our analysis of vNT, we see that the emphasized statement in the quotation is wrong. It is true that, given the quantum states admitted by the trace rule (1), which are always dispersive, II is enough to define quantities like $\mathcal{R}+\mathcal{S}$ by means of an operator $R+S$. However, since a theory that does admit dispersion-free states must reject $\mathbf{B}^{\prime}$ (even if only for the case of incompatible quantities and deterministic states), the theory cannot define a beable like $\mathcal{R}+\mathcal{S}$ by the operator $R+S$. A deterministic HVT that rejects $\mathbf{B}^{\prime}$ must reject II as well, so it cannot represent its beables by means of Hermitian operators. Thus, a viable deterministic HVT must significantly depart from the general structure of ordinary quantum mechanics.
} 
operators $U$ in $\mathcal{H}$, and expectation values of observables are given by $\langle R\rangle_{U}=\operatorname{Tr}(U R)$, where $R$ is Hermitian.

There are interesting differences, though. First, in GT, the result holds for $n$-dimensional Hilbert spaces with $n \geq 3$. The derived trace rule holds for 1- and 2-dimensional subspaces $A$, though, but as long as they are embedded in a 3-dimensional space. ${ }^{14} \mathrm{~A}$ more important difference is that GT makes use of sets of orthogonal projectors that add up to $I$ to represent possible measurement outcomes. Since these projectors mutually commute, the derivation of the trace rule in this theorem assumes a condition weaker than von Neumann's B', namely, the additivity of expectation values of commuting operators $\left\langle\sum P_{i}\right\rangle=\sum\left\langle P_{i}\right\rangle$. In other words, whereas both theorems prove that (1) determines the quantum states allowed and the expectation values (or measures), vNT proves it from $\mathbf{A}^{\prime}$, $\mathbf{B}^{\prime}, \mathbf{I}$ and II, whereas GT proves it from $\langle I\rangle=1$ and $\left\langle\sum P_{i}\right\rangle=\sum\left\langle P_{i}\right\rangle$.

The close connection between the meaning and aim of vNT and GT is not only a matter of conceptual analysis, there is also historical evidence for it. In a paper published five years before his seminal book, von Neumann (1927a) had already presented the essentials of the axiomatized formulation of quantum theory in the framework of Hilbert space. In a subsequent paper entitled Wahrscheinlichkeitstheoretischer Aufbau der Quantenmechanik (1927b), he complained that in his previous article he had uncritically assumed the Born rule and formulated its counterpart in Hilbert space: the trace rule (1). Von Neumann thought that given its relevance, (1) should be adopted in the theory not only on the basis of empirical adequacy - as it was in his first 1927 paper - but it should also be derived from basic formal principles in Hilbert space (see Duncan \& Janssen 2013, 246).

Von Neumann (1927b) accomplished that goal in the Aufbau paper, and the derivation of the trace rule he presented there is simply the first step in our review of vNT in the 1932 book (see Duncan \& Janssen 2013, 247-250). In the Aufbau paper, von Neumann also proved the corollary that if the density operator $U$ is homogeneous, it is a projector onto a unit vector. However, in 1927 he did not connect the derivation of (1), nor this corollary, with the possibility of an ignorance interpretation of the probabilities. In a word, the second paper of 1927 presents (the early version of) vNT only as a proof that from basic principles in Hilbert space the trace rule follows, regardless of issues about hidden variables. This is interesting because, seen in this way, vNT is obviously quite analogous to GT. But then in this reading we would hardly object against $\mathbf{B}^{\prime}$ along the lines of Hermann and Bell, especially if we consider von Neumann's rationale for it as a principle that allows to functionally define quantities like $\mathbf{f}(\mathcal{R}, \mathcal{S})$ when $\mathcal{R}$ and $\mathcal{S}$ are experimentally incompatible.

Now, if we further ask about the possibility of deterministic states and an ignorance interpretation of (1) for the quantum Hilbert space formalism, the second stage of vNT presented in 1932 answers both questions in the negative. It is then an unfortunate historical fact that vNT was misconceived as a general impossibility proof (correct or incorrect) of HVTs, rather than properly understood as a derivation of the trace rule from basic principles in Hilbert space, from which a no-go result for HVTs in Hilbert space follows as a corollary.

That vNT is not only a result about HVTs, but also a proof that quantum mechanical states in Hilbert space are always given by density operators, just like GT, has been rarely noticed in the

\footnotetext{
${ }^{14}$ Using positive operator valued measures (POVMs) instead of projectors, Paul Busch (2003) derived a proof of GT that holds also for 2-dimensional Hilbert spaces. Busch's is a valuable result, for it allows us to generalize everything we will say about Hermitian operators, on the basis of GT, to POVMs as well.
} 
philosophy and foundations literature. ${ }^{15}$ Another important connection between the theorems, that, as far as I know, has not been noticed at all, is that exactly the same restriction that vNT (rightly interpreted) imposes on HVTs can be straightforwardly derived from GT.

Recall our analysis of vNT in two steps. First a derivation of (1) from $\mathbf{A}^{\prime}, \mathbf{B}^{\prime}, \mathbf{I}$ and II, and then a proof that $\langle\mathcal{R}\rangle_{U}=\operatorname{Tr}(U R)$ does not admit dispersion-free states whereas it does admit dispersive homogeneous states, in which $\mathbf{A}^{\prime}, \mathbf{B}^{\prime}, \mathbf{I}$ and II are not used-only the definitions of dispersion-free states, homogeneous states, and some mathematical properties of Hilbert space are needed. Thus, it is easy to see that we can run the second step in vNT, from Gleason's derivation of (1), but this time the second step is not conditional on $\mathbf{B}^{\prime}$, but on the weaker premise $\left\langle\sum P_{i}\right\rangle=\sum\left\langle P_{i}\right\rangle$, so the HermannBell objection does not apply. Now, a most important issue here is that if we take the weaker premise $\left\langle\sum P_{i}\right\rangle=\sum\left\langle P_{i}\right\rangle$ as assuming that the projectors (and Hermitian operators in general) represent beables, then dispersion-free states do not exist and there are homogeneous states. That is, GT also shows the impossibility of embedding the quantum formalism in a HVT in Hilbert space.

If we consider Bub's reassessment of vNT, we have that GT establishes the very same result for HVTs. That is, if Hermitian operators represent physical properties of a system, and projectors represent yes-no propositions about values for those properties (according, most naturally, to $\left\langle\sum P_{i}\right\rangle=$ $\left.\Sigma\left\langle P_{i}\right\rangle\right)$, we obtain the trace rule (1), which we know does not admit dispersion-free states, but does admit homogeneous ones. In short, just like vNT, GT shows the unviability of HVTs that admit dispersion-free states and represent their beables in terms of Hermitian operators. According to both vNT and GT, if in a putative HVT Hermitian operators are upgraded from their role of conveying probability measures of measurement outcomes to representatives of properties, then dispersionfree states cannot be defined. Thus, viable HVTs need to go beyond Hilbert space and represent their beables in some other way.

After this analysis one might be tempted to state that Gleason proved the same result as von Neumann, but without invoking the problematic, or at least too strong, assumption $\mathbf{B}^{\prime}$. However, after our analysis of the justification for $\mathbf{B}^{\prime}$, I think a more accurate evaluation is the following. Von Neumann established the trace rule (1) by starting out from the condition that the full representative power of observables in Hilbert space is guaranteed by $\mathbf{B}^{\prime}$. It then turned out that HVTs in Hilbert space are not possible. Gleason did not assume this condition from the outset, but since his theorem also leads to the result that HVTs are not possible, B', which follows from the linearity of (1), holds for all allowed states (they are all dispersive). Then, the representative power of Hermitian operators in Hilbert space is guaranteed as a result in GT. In this sense, the theorems complement each other, and we see that $\mathbf{B}^{\prime}$ is not an unjustified assumption introduced by von Neumann. The proper lesson from critiques like Bell's and Hermann's is that $\mathbf{B}^{\prime}$ restricts the scope of vNT to Hilbert space, but it is precisely this restriction that makes the theorem so important for HVTs.

\section{KOCHEN \& SPECKER MEET VON NEUMANN AND GLEASON}

As we saw, in the KST, FUNC is imposed only on beables that are represented by operators that are the sum or the product of commuting operators, whereas in vNT, B' holds also for operators that

${ }^{15}$ To my knowledge, only Jammer (1974, 297), Giuntini \& Laudisa (2001), Busch (2003), and Bub (2010) have noticed the connection (but under different evaluations). 
are a linear combination of non-commuting operators. This difference, in the light of Bell's criticism, has led to a widespread opinion that the KST is much more relevant than vNT for HVTs (cf. Held 2018). We also saw that it is a common belief that the relevance of the KST is that it imposes some form of contextuality on the ontology of beables of quantum systems in HVTs.

We can now see that both these judgments are misconceptions. After Jammer's and Bub's clarification of vNT, and given our examination of GT as proving the same result on the basis of weaker premises, we can clearly see that the KST has a rather weak import on HVTs, and that it does not really impose any form of contextuality on the beables in such theories. As we will now show, a HVT that avoids vNT and GT, avoids the KST as well, but not conversely. Besides, since in HVTs that avoid vNT and GT beables are not represented by Hermitian operators, the KST has no relevance for the ontology of beables in such theories. ${ }^{16}$

\subsection{CONSTRAINING STRENGTH}

Let us briefly recall Redhead's analysis of the KST. It starts out from a characterization of a HVT in Hilbert space in terms of i) quantum systems always have definite values for all beables independently of measurements; ii) every Hermitian operator represents a beable; and iii) every beable is represented by one Hermitian operator. A HVT in which i), ii) and iii) all hold is compelled to a FUNC-constrained complete and consistent assignment of beable values. The KST shows that such an assignment is impossible, so it forces HVTs to drop one of the assumptions i), ii), and iii).

By dropping i) we get contextuality of the form a): for some beables, a system has definite values only relative to measurement contexts. By dropping ii), we get contextuality of type b): even though $A, B$ and $C$ all represent beables and $C=f(A)=g(B), f(A)$ represents a beable, but $g(B)$ does not, so whether $C$ represents a beable or not depends on the measurement context. By dropping iii) we are led to contextuality of the form c): $f(A)$ and $g(B)$ represent different beables, so which beable $C$ represents depends on the measurement context. All these strategies concern the ontology of the beables in the putative HVT.

On the other hand, Bub and Jammer have clarified that the true significance of vNT is that it rules out HVTs in which beables are represented by Hermitian operators, or more generally, that HVTs cannot be Hilbert space theories. Furthermore, we have shown that GT proves the same result on stronger grounds. Let us then consider what comes of the three forms of contextuality identified by Redhead (1987), in the light of vNT and GT.

Both in b) and c), although not bijectively, the putative theory represents all its beables in terms of Hermitian operators, so expectation values are always given by the trace rule (1). In other words, HVTs that are contextual as in b) and c) are certainly Hilbert space theories. Thus, after the reviewed reassessment of the relevance of vNT and GT for HVTs, we get that such theories fall prey to vNT and GT. In short, HVTs characterized by [i) $\wedge \neg$ ii) $\wedge$ iii)] (contextuality b)), or by [i) $\wedge$ ii) $\wedge \neg$ iii)] (contextuality c)), are unviable by vNT and GT.

In theories that take the a) avenue, both ii) and iii) hold. Thus, a HVT of the type a) is obviously a Hilbert space theory in which Hermitian operators bijectively represent its beables, and (1) conveys

\footnotetext{
${ }^{16}$ My deflationary interpretation of the KST is circumscribed to the ontology of HVTs. I do not mean that the theorem is not important in other contexts (quantum information and quantum logic, for example).
} 
the expectation values. We get then the same result. Ontologically contextual HVTs that go around the KST by strategy a), i.e., in which $[\neg$ i) $\wedge$ ii) $\wedge$ iii)], are also unviable by vNT and GT.

It turns out then that the type of theories on which the KST imposes a contextual ontology of its beables are all Hilbert space theories, so their beables are always represented by Hermitian operators. Thus, the HVTs that are doomed to be ontologically contextual according to the standard reading of the KST are rather uninteresting: they belong to the class of theories that is already ruled out by vNT and GT.

On the other hand, in a HVT that avoids both vNT and GT, the beables are not represented by Hermitian operators. Hence, neither ii) nor iii) can hold in such a theory, and the KST no-go result is sidestepped. The upshot for our discussion is thus that a HVT that avoids vNT and GT, avoids the KST as well, but not conversely. ${ }^{17}$ The upshot is then that the comparative evaluation, after Bell's analysis, that the KST is more relevant than vNT is upside down. Since a viable HVT is to observe the constraints imposed by all three theorems, the restriction imposed by vNT and GT is actually stronger and more fundamental than the one imposed by the KST.

\subsection{CONTEXTUALITY DEFLATED}

Given this diagnosis, we may now ask what is the precise conceptual import of the KST for HVTs. In Redhead's (1987) analysis, a HVT in Hilbert space can only retain i) on the price of installing contextuality for its beables in the form of b) or c). But as we just saw, Hilbert space HVTs are ruled out by vNT and GT anyway. Let us then consider a HVT that does avoid both vNT and GT. In such a theory, neither ii) nor iii) holds, so, in principle, it can retain i) without making any contextuality concessions regarding the ontology of its beables. The spirit of HVTs expressed by i) is not threatened in any sense by the KST when we consider the lesson to be learned from vNT and GT.

Let us elaborate. We saw in section 2.2 that we can take the KST in strictly mathematical terms. The impossibility of assigning completely and consistently the number 1 to one ray in each orthogonal triad and the number 0 to the remaining rays still holds, of course. Now, in a viable HVT the trace rule (1) must hold as a matter of empirical adequacy, so some operational role must be assigned to Hermitian operators in the theory. That is, Hermitian operators will certainly work as $o b-$ servables in that theory, in the sense of representing numerical outcomes of experiments, but not possessed beable values.

Now, that a FUNC-constrained complete and consistent assignment of (eigen)values for observables is not possible does not mean that there must be some type of contextuality affecting the beables in the putative HVT. If Hermitian operators are deprived of the role of representing beables, the KST imposes a contextuality constraint that is only phenomenological on such theories. That is, in viable HVTs, what is impossible by the KST is simply a FUNC-constrained complete and consistent assignment of numerical outcomes of experiments given by eigenvalues of Hermitian operators, so the weak counterfactual reading I presented in section 2.2 above captures the right import of the KST for

\footnotetext{
${ }^{17}$ As a matter of logic, one could still conceive a deterministic HVT in which i) holds, in which Hermitian operators do not represent beables (so that ii) and iii) do not hold), but in which other tenets still lead to a FUNC-constrained complete and consistent assignment of eigenvalues to projectors (as we will see below, empirical adequacy requires that Hermitian operators must still represent experimental outcomes). However, it is hard to conceive a plausible physical motivation for such a convoluted HVT. Interestingly, in section 6 we will see that this highly implausible HVT does not necessarily fall prey to the KST.
} 
HVTs. That is, in viable HVTs, given a projector-observable $P=f Q=g Q^{\prime}$, such that $\left[Q, Q^{\prime}\right] \neq 0$, if in a $P$-experiment via a $Q$-experiment we get the (eigen)value 1 , in a $P$-experiment via a $Q^{\prime}$-experiment we would have obtained an (eigen)value 0 . But in both cases, the obtained (eigen)values are only numerical results of experiments, not beable values.

Thus, if we consider vNT and GT, the KST constraint affects only the phenomenology of HVTs, not their ontology. In simple words, for HVTs that observe vNT and GT constraints, the KST enforces that numerical outcomes of experiments must depend on the measurement context, but that does not mean that there must be something contextual at the level of beables ontology. If we still decide to use the term "contextuality" to characterize this formulation of the meaning of the KST, it cannot have the ontological import that is usually associated with it. If Hermitian operators have an operational role other than representing beables (as it is enforced by vNT and GT), and we consider that $Q$ - and $Q^{\prime}$-measurements require different experimental setups and involve different physical interactions between system and experimental device, there is nothing strange or peculiarly quantum about the phenomenological contextuality that the KST imposes. Actually, given the role that Hermitian operators play as representatives of numerical outcomes in experiments, this form of contextuality is quite natural and expected.

Actually, there is an extant example of all of this: Bohm's theory. In Bohmian mechanics, systems are described by $(\psi, Q)$, where $\psi=R^{e i s / \hbar}$ is the wavefunction, and $Q$ is the configuration of the particles, given by the positions $\mathbf{x}$ of all the particles in the system. Momentum is given by $\mathbf{p}=\nabla S(\mathbf{x}) / \mathrm{m}$. The fundamental beable is of course $\mathbf{x}$, and other properties are given by functions of positions and momentum, not by Hermitian operators. For example, the total energy is given by $E=\frac{(\nabla S)^{2}}{2 m}+W+$ $V$, where $W$ and $V$ are the quantum and the classical potentials, respectively. Since we only have access to the statistical distribution of the particles, given by $R^{2}=|\psi|^{2}$, predictions are probabilistic, and they are numerically equal to the expectation values given by Hermitian operators and the trace rule (1). In our example of energy, it holds that

$$
\langle E\rangle_{\psi}=\int R^{2}\left[\frac{(\nabla S)^{2}}{2 m}+W+V\right] \mathrm{d}^{3} \mathrm{x}=\int \psi^{*}\left[-\left(\frac{\hbar^{2}}{2 m}\right) \nabla^{2}+V\right] \psi \mathrm{d}^{3} x=\langle H\rangle_{\psi}
$$

In the literature on Bohmian mechanics it has been clear for a while that Hermitian operators are not representatives of beables. Rather, they are "book-keeping devices for [...] wavefunction statistics" (Dürr \& Teufel 2009, 228). In a word, in Bohm's theory Hermitian operators map the composite of measured system plus experimental apparatus, after they have interacted, to probabilistically distributed numerical (eigen)values - see (Daumer et al. 1997) and (Dürr, Goldstein \& Zanghì 2004). Then, if an operator $O$ is a function of two non-commuting operators, associated to two incompatible experimental procedures, that the obtained eigenvalue for an $O$-measurement depends on the experimental context is quite natural, and has nothing to do with some bizarre ontological contextuality of beables - the interactions in the experimental procedures are different, that is all. An important point for us is that the analysis of the role of Hermitian operators presented in (Daumer et al. 1997) and (Dürr, Goldstein \& Zanghì 2004) is quite general. That is, it does not refer to peculiar features of Bohm's theory, so the same is expected in any viable HVT that assumes principle i). 
A concrete illustration of how contextuality works in Bohmian mechanics is presented in Bub (1997, 165-169) (cf. Pagonis \& Clifton 1995). The squared-spin operator in the $z$-direction $S_{z}^{2}$ is a function of two non-commuting operators $H$ and $H^{\prime}$, that is, $S_{z}^{2}=H^{2}=H^{\prime 2}$, where $H=S_{x}^{2}-S_{y}^{2}$ and $H^{\prime}=$ $S_{x^{\prime}}^{2}-S_{y^{\prime}}^{2}\left(x, y, z\right.$ and $x^{\prime}, y^{\prime}, z$ are two different triads of orthogonal directions). Since an $H$-experiment and an $H^{\prime}$-experiment are incompatible, that the $S_{z}^{2}$-value obtained depends on whether an $H$ or an $H^{\prime}$-experiment is performed is totally natural if we keep in mind that Hermitian operators map the composite of measured systems plus experimental apparatus, after they have interacted, to probabilistically distributed numerical (eigen)values. ${ }^{18}$

As it is clear, this form of contextuality, which is an instance of the weaker physical reading of the relevance of the KST, has nothing to do with the ontology of the beables of systems in a HVT like Bohmian mechanics. At an ontological level, position and the rest of Bohmian beables are not contextual: this theory is not contextual in the form of a), b) or c). As Clifton \& Pagonis conclude, Bohmian contextuality is "hardly the sort of contextualism to write home about" $(1995,290)$. After our examination of the constraints on HVTs imposed by vNT and GT, and our reassessment of the relevance of the KST for hidden variable theories in the light of such constraints, we can see that this is not a contingent quality of Bohmian mechanics, but an expected feature in any viable HVT. ${ }^{19}$

\subsection{BELL AND GLEASON}

In the same article in which Bell (1966) presented his criticism of vNT, he derived a result equivalent to the KST as a corollary to GT. A simple reconstruction of Bell's reasoning is the following. We can take $\mu(A)=\operatorname{Tr}\left(U P_{A}\right)$ as a map $\mu^{\prime}$ from each point in the surface of the unit sphere in a 3dimensional Hilbert space onto [1,0]. According to $\mu(A)=\operatorname{Tr}\left(U P_{A}\right)$, the map $\mu^{\prime}$ is continuous, in the sense that sufficiently small changes in positions on the sphere produce small changes in the corresponding probabilities. ${ }^{20}$ If we assume a HVT characterized by i), ii), and iii), in a FUNC-constrained complete and consistent assignment of values to pairs of antipodal points in the sphere (determined by projectors), the points in each pair should be assigned a 1 or a 0 , and both values must occur. Thus, it must hold that for two arbitrarily close points, one gets the value 1, and the other the value 0 . But since $\mu^{\prime}$ is continuous, the FUNC-constrained and complete assignment leads to a contradiction.

Bell was quick to realize that this is not an absolute proof of the impossibility of HVTs: it does rule out theories that impose a FUNC-constrained complete and consistent assignment of $1 \mathrm{~s}$ and $0 \mathrm{~s}$ to all the projectors onto the rays that determine antipodal points in the surface of the unit sphere, but contextual assignments are still possible. Besides, he was explicit in that this result is not puzzling if we consider that the impossible assignment requires that a projector-measurement must yield the same result independently of what other experiments are made simultaneously:

\footnotetext{
${ }^{18}$ Bohm himself was aware of the role that Hermitian operators play in his theory: "the measurement of an "observable" is not really a measurement of any physical property belonging to the observed system alone. Instead, the value of an "observable" measures only an incompletely predictable and controllable potentiality belonging just as much to the measuring apparatus as to the observed system itself" (Bohm 1952, 183).

${ }_{19}$ That in deterministic HVTs the beables cannot be represented by Hermitian operators implies that Bohmian mechanics is not an interpretation of quantum mechanics but a rival theory, see Acuña (2019).

${ }^{20}$ Notice that the continuity of the map $\mu^{\prime}$ also holds in von Neumann's derivation of the trace rule (1), which in turn means that Bell's corollary to GT can also be derived from vNT.
} 
The danger in fact was not in the explicit but in the implicit assumptions. It was tacitly assumed that measurement of an observable must yield the same value independently of what other measurements may be made simultaneously. Thus as well as $P_{\left(\Phi_{3}\right)}$ say, one might measure either $P_{\left(\Phi_{2}\right)}$ or $P_{\left(\Psi_{2}\right)}$, where $\Phi_{2}$ and $\Psi_{2}$ are orthogonal to $\Phi_{3}$ but not to one another. These different possibilities require different experimental arrangements; there is no a priori reason to believe that the results for $P_{\left(\Phi_{3}\right)}$ should be the same. The result of an observation may reasonably depend not only on the state of the system (including hidden variables) but also on the complete disposition of the apparatus. (Bell 1966, 451; my emphasis)

In this passage, the evaluation of the relevance of the KST that Bell defends as reasonable comes close to the one that we are proposing here. Now, another way to make our point is that this interpretation, which Bell characterizes as plausible, is actually enforced when we consider the stronger constraint imposed on HVTs by vNT and GT. Unfortunately, Bell's criticism of vNT precluded him to see that. Once again, in HVTs operators must have the operational role of being representatives of numerical outcomes of experiments, but they cannot be representatives of beables-Bell did not notice that this is not only reasonable, but commanded by vNT (and GT). Then, in the light of this command, the KST forces HVTs to yield numerical outcomes of experiments in a context-dependent way, but this is hardly surprising, and there is nothing peculiarly quantum about it.

In other words, Bell's own contextuality result offers us one more way to illustrate our point. The reader may have noticed the similarity between Bell's contextuality proof and von Neumann's demonstration that the trace rule does not allow dispersion-free states (see section 3.1 above), but in close inspection they are very different. Von Neumann (and Gleason) proved that if we assume that beables are represented by Hermitian operators, states can only be given by the density operators $U$ in the trace rule (1). In turn, (1) determines that the map $\mu^{\prime}$ from measures to the points on the surface of the unit sphere must be continuous, which means that, for hypothetical dispersion-free states, all the points along a continuous path between two points should be assigned with the same number: either 1 or 0 . But then it should be the case that $U=I$ or $U=0$, but this is impossible since $\operatorname{Tr} U=1$. On the other hand, Bell uses the same continuity enforced by (1) to show that a non-contextual distribution of $1 \mathrm{~s}$ and $0 \mathrm{~s}$ for the points on the surface of the unit sphere is impossible. Hence, since dispersion-free states must assign either 1s or 0s to the points on the surface, it follows that if there are such states, the assignment they induce must be contextual. But since by vNT and GT the possibility of such states requires that there cannot be a representative link between Hermitian operators and beables, the contextuality in Bell's corollary (and in the KST) does not affect the beables. ${ }^{21}$

\section{THE MEYER-KENT-CLIFTON ARGUMENT}

\footnotetext{
${ }^{21}$ After these remarks we can see GT in all its remarkable strength. First, it shows that if measurement outcomes are given by projectors, measures over those outcomes are always given by the trace rule (1). Second, a "von Neumann corollary" follows that deterministic HVTs in Hilbert space are ruled out. Thirdly, a "KS-Bell corollary" follows that a non-contextual assignment of eigenvalues to projectors is not possible. After what we said in section 4, we get the interesting result that $v N T$ is equally strong: both corollaries can also be obtained from von Neumann's derivation of (1). As we saw, the difference lies on the premises: $\langle I\rangle=1$ and $\left\langle\sum P_{i}\right\rangle=\sum\left\langle P_{i}\right\rangle$ in GT; $\mathbf{A}^{\prime}, \mathbf{B}^{\prime}, \mathbf{I}$ and II in vNT.
} 
The analysis presented allows us to differentiate my proposal from an interesting argument that is apparently similar in spirit-it claims to have "nullified" the significance of the KST-and to reevaluate the significance of that argument in new light.

Recall that the KST, in the Kochen-Specker (1967) formulation, proceeds by identifying a set of rays in $\mathcal{H}^{3}$ for which a complete and consistent FUNC-constrained assignment is impossible. Following the jargon in the literature, let us call such a set a KS-uncolorable set. ${ }^{22}$ On the other hand, we saw that in Bell's (1966) proof the impossibility of the KS-coloring is derived from the continuity of the mapping $\mu^{\prime}$. David Meyer (1999) proved that if in order to determine the set of rays (that correspond to the projectors of a physical system) to be KS-colored in a 3-dimensional Hilbert space we use rational coordinates, instead of real (or complex) coordinates, KS-colorings are possible. Adrian Kent (1999) extended this result to Hilbert spaces $\mathcal{H}^{n}$ of any dimensions.

The sense in which these results nullify the KST, in the Kochen-Specker (1967) formulation, is that for any KS-uncolorable set of rays, there is a KS-colorable set in which the rays are arbitrarily close to the rays in the former set. The nullification of Bell's (1967) proof consists in that the KS-colorable set is dense, but the corresponding mapping $v$ from each point in the unit sphere (determined by rational-coordinates rays) to 1 or 0 is not continuous. The physical motivation for such a construction, Meyer (1999) states, is that measurements are always of finite precision.

Rob Clifton \& Adrian Kent (2000) (see also Barrett \& Kent 2004) have shown that it is possible to construct a HVT, empirically indistinguishable from ordinary quantum theory, that yields a KScolored dense set of projectors $\mathcal{P}$ for a physical system. Let $\mathcal{C}$ be the set of all the decompositions of the identity operator $I$ in terms of orthogonal projectors in $\mathcal{H}^{n}$. Kent's result implies that $\mathcal{P}$ is dense in the set of all projectors in $\mathcal{H}^{n}$, and also that the set $\mathcal{D}$ of all projective decompositions of $I$ given by the projectors in $\mathcal{P}$ is dense in $\mathcal{C}$. Now, let us assume that every measurement corresponds to a decomposition of the identity operator $I$ in terms of orthogonal projectors. However, given finite precision, we can stipulate that not every projective decomposition of $I$ in $\mathcal{C}$ corresponds to a possible measurement, but only the ones in $\mathcal{D}$. Thus, in our putative HVT, "the result of any measurement is determined by hidden variables that assign a definite value to each operator in $\mathcal{P}$ in a non-contextual manner" (Barret \& Kent 2004, 158).23

Now, given a certain measurement, we may ask which of the elements in $\mathcal{D}$ our HVT determines that the measurement corresponds to. Clifton \& Kent (2000) introduce an algorithm to answer this question, which is further elaborated in Barret \& Kent (2004):

Consider some ordering $\left\{d^{1}, d^{2}, \ldots\right\}$ of the countable set $\mathcal{D}$. Let $\varepsilon$ be a parameter much smaller than the precision attainable in any current or foreseeable experiment. More precisely, $\varepsilon$ is sufficiently small that it will be impossible to tell from the outcome statistics if a measurement attempts to measure a decomposition $d=\left\{P_{1}, \ldots, P_{n}\right\}$ and actually measures a decomposition $d^{\prime}=\left\{P_{1}^{\prime}, \ldots, P_{n}^{\prime}\right\}$ provided $\mid P_{i}-$

\footnotetext{
${ }^{22}$ It is usual to describe a complete and consistent FUNC-constrained assignment as an assignment of colors to the surface of the unit sphere in an $n$-dimensional Hilbert space such that for $n$ pairs of antipodal points determined by mutually orthogonal rays, one pair is assigned one color (e.g., green), and the rest with another color (e.g., red).

${ }^{23}$ As Held (2018, section 6) points out, if the HVT assigns a physically meaningful value (as possessed beable values) to the projectors that constitute KS-uncolorable sets, the theory would still be ontologically contextual. But if we cut such projectors from the ontology, then the resulting HVT would be rather bizarre. In the case of spin, for example, the theory would state that not every one of the continuous different directions in physical space determines a spinbeable and a spin-measurement, or that there are not continuously many directions in physical space. For a critical assessment of the MKC argument based on the same issue, see Appleby (2005).
} 
$P_{i}^{\prime} \mid<\varepsilon$ for all $i$. Suppose now we design a quantum experiment which would, if quantum theory were precisely correct, measure the projective decomposition $d$. [...] We could imagine that the hidden variable theory uses the following algorithm: first, it identifies the first decomposition $d^{i}=\left\{P_{1}^{i}, \ldots, P_{n}^{i}\right\}$ in the sequence such that $\left|P_{j}-P_{j}^{i}\right|<\varepsilon$ for all $j$ from 1 to $n$. Then, it reports the outcome of the experiment as that defined by the hidden variables for $d^{i}$ : in other words, it reports outcome $j$ if the hidden variable theory ascribes value 1 to $P_{j}^{i}$ (and hence 0 to the other projectors in $d$ ). (Barret \& Kent 2004, 158159)

Let us now assess the MKC argument from the point of view of the KST in the light of vNT and GT. First notice that the algorithm obviously presupposes that the predictions in the HVT are given by the trace rule (1) (cf. Clifton \& Kent 2000, 2106). Now, that the mapping $v$ (given by the algorithm on the basis of the trace rule) from the points in the unit sphere (determined by the projectors in the dense set $\mathcal{P}$ ) to 1 or 0 is not a continuous function does not alter the fact that (1) does not admit dispersion-free states, but admits homogeneous ones. Although it is clear that Clifton \& Kent (2000) and Barrett \& Kent (2004) have HVTs in Hilbert space in mind (so that projectors represent beables), we now know that such theories are impossible.

Then, what the MKC argument proves is that a there can be a HVT that assigns numerical (eigen)values to experimental outcomes in a complete and consistent FUNC-constrained (non-contextual) manner, as long as the Hermitian operators that describe experimental outcomes are given by the dense sets $\mathcal{P}$ and $\mathcal{D}$. In other words, the argument shows that there can be HVTs, empirically indistinguishable from ordinary quantum mechanics, which, despite the KST, are not forced to be phenomenologically contextual in the sense described in section 5.2. However, we now know that, despite the KST, by vNT and GT there was no ontological contextuality to be nullified in the first place. That is, considering the restriction that vNT and GT impose on HVTs, the (phenomenological) non-contextuality that the MKC reinstates has no ontological import. Considering that phenomenological contextuality is quite natural and expected in HVTs that avoid vNT and GT, the upshot is that there is no ontological motivation for the non-contextual HVTs that the MKC argument shows to be possible. ${ }^{24}$

\section{CONCLUDING SUMMARY}

According to philosophy of physics folklore, the relevance of vNT is quite weak and uninteresting. After Bell's and Hermann's criticisms that made clear that the theorem is not an impossibility proof, it is a widespread belief that its only significance is that it rules out an uninteresting class of HVTs.

\footnotetext{
${ }^{24}$ Phenomenologically non-contextual theories that avoid vNT and GT would be highly implausible (cf. fn. 17). As we saw in section 5.2, phenomenological contextuality is an expected and natural feature in HVTs in which Hermitian operators denote observables, but not beables, so there is no reasonable motivation for a HVT to be phenomenologically non-contextual. Furthermore, Appleby (2002) shows that the HVTs in the MKC argument have a very strange property. For entangled states, the choice of a determinate direction for a spin-measurement on Alice's particles determines the directions that admit physically possible spin-measurements on Bob's particles (cf. fn. 23) i.e., Alice's measurement context determines the physically well-defined quantities in Bob's lab. When we consider that in the phenomenologically non-contextual HVTs that the MKC argument shows possible Hermitian operators represent observables, but not beables, this feature becomes a bizarre form of non-locality that goes beyond the nonlocality enforced by Bell's theorem. Anyhow, the MKC mathematical result that there exist dense KS-colorable sets in Hilbert spaces of any dimensionality is certainly interesting, and it might be significant for issues in quantum information and quantum logic (cf. fn. 16).
} 
On the other hand, the standard reading of the KST is that it forces HVTs to make a huge concession in their basic spirit and motivation, for it allegedly shows that in such theories there will be some properties that are ontologically dependent on the measurement context.

As we have seen, both these official stances are misconceptions. As Jammer, Bub and Dieks have convincingly argued, vNT shows that HVTs cannot be Hilbert space theories, or, more precisely, that a viable HVT cannot represent its beables by means of Hermitian operators. As I have shown, the very same result can be derived from Gleason's unquestionable theorem, on the basis of weaker premises that are not subject to the criticism that Grete Hermann and John Bell levelled against vNT.

On the other hand, a careful analysis shows that the interpretation that the KST imposes an ontological contextuality constraint on the beables in HVTs crucially presupposes that such beables are represented by Hermitian operators, or, in other words, that the HVTs that are condemned to be ontologically contextual are Hilbert space theories. Thus, if we interpret the KST in the light of a correct reading of vNT, and also in the light of GT, it turns out that the HVTs in which beables are ontologically contextual are unviable. Furthermore, viable HVTs that avoid both vNT and GT also naturally avoid the ontological contextuality result of the KST. The upshot is that vNT and GT have a much more fundamental relevance for HVTs than the KST.

The precise import that the mathematical result obtained by Kochen \& Specker has for HVTs is that a FUNC-constrained complete and consistent assignment of (eigen)values to Hermitian operators-as-observables is not possible (unless we restrict our operators-as-observables to the dense sets $\mathcal{P}$ and $\mathcal{D}$ in the MKC argument). That is, since by vNT and GT such operators cannot be representative of the theory's beables, the contextuality at issue, if we still decide to use this term, does not affect their ontology. Given that Hermitian operators must still have an operational meaning in HVTs, the import of the KST is only phenomenological. This is actually a well-known feature in Bohm's theory, so now we understand that it is not an accidental quality of Bohmian mechanics, but an expected characteristic - enforced, bottom-line, by vNT and GT - in any viable HVT.

\section{ACKNOWLEDGMENTS}

I thank Jeff Bub and three anonymous referees for their comments on an earlier version of this manuscript. I also thank Michel Janssen for calling my attention to von Neumann's 1927 papers. This work was financially supported by FONDECYT grant 11170608.

\section{REFERENCES}

Acuña, P. (2019). Charting the Landscape of Interpretation, Theory Rivalry, and Underdetermination in Quantum Mechanics. Synthese, https://doi.org/10.1007/s11229-01902159-z.

Appleby, D. M. (2002). Existential Contextuality and the Models of Meyer, Kent and Clifton. Physical review $A, 65,022105$. 
Appleby, D. M. (2005). The Bell-Kochen-Specker Theorem. Studies in History and Philosophy of Modern Physics, 36, 1-28.

Aravind, P. K. (1999). Impossible Colorings and Bell's Theorem. Physics Letters A, 262, 282-286.

Bacciagaluppi, G., \& Crull, E. (2009). Heisenberg (and Schrödinger, and Pauli) on Hidden Variables. Studies in History and Philosophy of Modern Physics, 40, 374-382.

Bacciagaluppi, G., \& Valentini, A. (2009). Quantum Theory at the Crossroads: reconsidering the 1927 Solvay Conference. Cambridge: Cambridge University Press.

Barrett, J., \& Kent, A. (2004). Non-Contextuality, Finite Precision Measurement and the KochenSpecker Theorem. Studies in History and Philosophy of Modern Physics, 35, 151-176.

Bell, J. (1966). On the Problem of Hidden Variables in Quantum Mechanics. Reviews of Modern Physics, 38, 447-452.

Belousek, D. (1996). Einstein's 1927 Unpublished Hidden Variables Theory: its background, context, and significance. Studies in History and Philosophy of Modern Physics, 27, 437-461.

Bohm, D. (1952). A suggested interpretation of the quantum theory in terms of "hidden" variables I-II. Physical Review, 85, 166-193.

Born, M., Heisenberg, W., \& Jordan, P. (1926). Zur Quantenmechanik II. Zeitschrift für Physik, 35, 557615.

Bub, J. (1997). Interpreting the Quantum World. Cambridge: Cambridge University Press.

Bub, J. (2010). Von Neumann's no 'No Hidden Variables' Proof: a re-appraisal. Foundations of Physics, 40, 1333-1340.

Bub, J. (2011). Is von Neumann's "No Hidden Variables" Proof Silly? In H. Halvorson (Ed.), Deep Beauty: understanding the quantum world through mathematical innovation (pp. 393-407). Cambridge: Cambridge University Press.

Busch, P. (2003). Quantum States and Generalized Observables: a simple proof of Gleason's Theorem. Physical Review Letters, 91, 120403.

Clifton, R., \& Kent, A. (2000). Simluating Quantum Mechanics by Non-Contextual Hidden Variables. Proceedings of the Royal Society of London A, 456, 2101-2114.

Cushing, J. (1994). Quantum Mechanics: historical contingency and the Copenhagen hegemony. Chicago: The University of Chicago Press.

Daumer, M., Dürr, D., Goldstein, S., \& Zanghì, N. (1997). Naive Realism about Operators. Erkenntnis, 45, 379-397.

Dieks, D. (2017). Von Neumann's Impossibility Proof: mathematics in the service of rhetorics. Studies in History and Philosophy of Modern Physics, 60, 136-148.

Duncan, A., \& Janssen, M. (2013). (Never) Mind your p's and q's: von Neumann versus Jordan on the foundations of quantum mechanics. The European Physical Journal H, 38, 175-259. 
Dürr, D., \& Teufel, S. (2009). Bohmian Mechanics: the physics and mathematics of quantum theory. Springer.

Dürr, D., Goldstein, S., \& Zanghì, N. (2004). Quantum Equilibrium and the Role of Operators as Observables in Quantum Theory. Journal of Statistical Physics, 116, 959-1055.

Giuntini, R., \& Laudisa, F. (2001). The Impossible Causality: the no hidden variables theorem of John von Neumann. In M. Rédei, \& M. Stöltzner (Eds.), John von Neumann and the Foundations of Quantum Physics (pp. 173-188). Kluwer.

Gleason, A. (1957). Measures on the Closed Subspaces of a Hilbert Space. Journal of Mathematics and Mechanics, 6, 885-893.

Held, C. (2018). The Kochen-Specker Theorem. In The Stanford Encyclopedia of Philosophy, URL = $<$ https://plato.stanford.edu/archives/spr2018/entries/kochen-specker/>.

Hermann, G. (2016). Natural-Philosophical Foundations of Quantum Mechanics. In G. Bacciagaluppi, \& E. Crull (Eds.), Grete Hermann: between physics and philosophy (pp. 239-278). Springer.

Holland, P. (2005). What's Wrong with Einstein's 1927 Hidden-Variable Interpretation of Quantum Mechanics? Foundations of Physics, 35, 177-196.

Jammer, M. (1974). The Philosophy of Quantum Mechanics. New York: John Wiley \& Sons.

Kent, A. (1999). Noncontextual Hidden Variables and Physical Measurements. Physical Review Letters, 83, 3755-3757.

Kochen, S., \& Specker, E. (1967). The Problem of Hidden Variables in Quantum Mechanics. Journal of Mathematics and Mechanics, 17, 59-87.

Mermin, N. D., \& Schack, R. (2018). Homer Nodded: von Neumann's surprising oversight. Foundations of Physics, 48, 1007-1020.

Myrvold, W. (2003). On some Early Objections to Bohm's Theory. International Studies on the Philosophy of Science, 17, 7-24.

Pagonis, C., \& Clifton, R. (1995). Unremarkable Contextualism: dispositions in the Bohm theory. Foundations of Physics, 25, 281-296.

Peres, A. (1993). Two Simple Proofs of the Kochen-Specker Theorem. Journal of Physics A, 24, L1758.

Redhead, M. (1987). Incompleteness, Nonlocality and Realism: a prolegomenon to the philosophy of quantum mechanics. Oxford: Clarendon Press.

Schrödinger, E. (1926). An Undulatory Theory of the Mechanics of Atoms and Molecules. The Physical Review, 28, 1049-1070.

von Neumann, J. (1927a). Mathematische Begründung der Quantenmechanik. Nachrichten von der Gesellschaft der Wissenschaften zu Göttingen, Mathematisch-Physikalische Klasse, 1927, 1-57. 
von Neumann, J. (1927b). Wahrscheinlichkeitstheoretischer Aufbau der Quantenmechanik. Nachrichten von der Gesellschaft der Wissenschaften zu Göttingen, Mathematisch-Physikalische Klasse, 1927, 245-272.

von Neumann, J. (1955). Mathematical Foundations of Quantum Mechanics. Princeton: Princeton University Press.

Whitaker, A. (1996). Einstein, Bohr, and the Quantum Dilemma. Cambridge: Cambridge University Press. 